\title{
Abstract Syntax Notation X (ASN.X) Representation of Encoding Instructions for the Generic String Encoding Rules (GSER)
}

\section{Status of This Memo}

This memo defines an Experimental Protocol for the Internet community. It does not specify an Internet standard of any kind. Discussion and suggestions for improvement are requested. Distribution of this memo is unlimited.

Copyright Notice

Copyright (C) The IETF Trust (2007).

Abstract

Abstract Syntax Notation X (ASN.X) is an Extensible Markup Language (XML) representation for Abstract Syntax Notation One (ASN.1) specifications. This document specifies the ASN.X representation of encoding instructions for the Generic String Encoding Rules (GSER).

Table of Contents

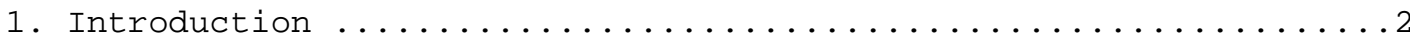

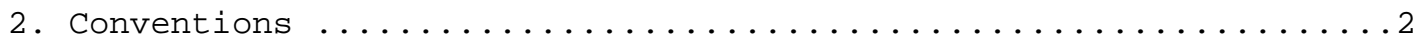

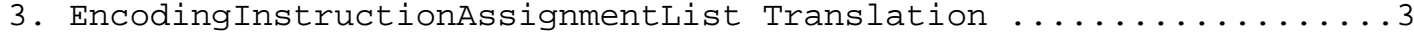

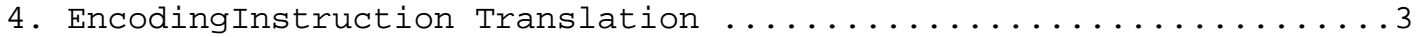

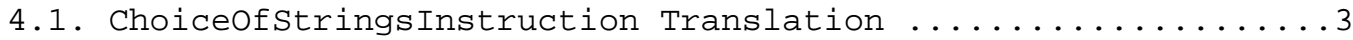

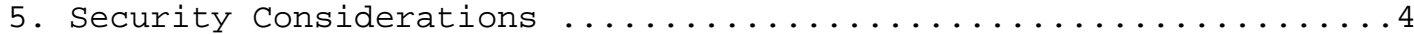

6. Normative References ........................... Appendix A. ASN.1 for GSER Encoding Instruction Notation .......6 Appendix B. ASN.X for GSER Encoding Instruction Notation .........7 
1. Introduction

Abstract Syntax Notation X (ASN.X) [ASN.X] is an Extensible Markup Language (XML) [XML10] [XML11] representation for Abstract Syntax Notation One (ASN.1) [X.680] specifications. The ASN.X representation for the ASN.1 basic notation [X.680] [X.680-1] is described elsewhere [ASN.X].

The grammar of ASN.1 permits the application of encoding instructions [X.680-1], through type prefixes and encoding control sections, that modify how abstract values are encoded by nominated encoding rules.

The generic notation for type prefixes and encoding control sections is defined by the ASN.1 basic notation; however, the notation for specific encoding instructions, i.e., the EncodingInstruction and EncodingInstructionAssignmentList productions of the notation, are defined separately for each set of encoding rules using encoding instructions. This document specifies the ASN.X representation for EncodingInstructionAssignmentList and EncodingInstruction as they are defined for the Generic String Encoding Rules (GSER) [GSER] [GSEREI] •

ASN.X is defined in terms of rules for translating from an ASN.1 specification. This does not preclude an ASN.X module being written directly without a pre-existing ASN.1 module; however, such an ASN.X module is considered valid if and only if there exists, in principle, an ASN.1 module that when translated would yield the ASN.X module.

By design, an ASN.X module is also the Robust XML Encoding Rules (RXER) [RXER] encoding of an ASN.1 value. The ASN.1 type definitions for such values, insofar as they pertain to the ASN.1 basic notation, are provided elsewhere [ASN.X]; however, this document provides the ASN.1 type definitions for representing GSER encoding instructions as abstract values. These definitions appear in Appendix A.

The ASN.X translation of the ASN.1 module in Appendix A is presented in Appendix B.

The General Considerations of the specification for A.SN.X [ASN.X] also apply here.

2. Conventions

The key words "MUST", "MUST NOT", "REQUIRED", "SHALL", "SHALL NOT", "SHOULD", "SHOULD NOT", "RECOMMENDED", and "MAY" in this document are to be interpreted as described in BCP 14, RFC 2119 [BCP14]. The key word "OPTIONAL" is exclusively used with its ASN.1 meaning. 
A reference to an ASN.1 production [X.680] (e.g., Type, NamedType) is a reference to the text in an ASN.1 specification corresponding to that production.

The description of the translation of GSER encoding instructions into ASN.X makes use of definitions from the XML Information set (Infoset) [INFOSET]. In particular, information item property names follow the Infoset convention of being shown in square brackets, e.g.,

[local name]. Literal values of Infoset properties are enclosed in double quotes; however, the double quotes are not part of the property values. In the sections that follow, "information item" will be abbreviated to "item", e.g., "element information item" is abbreviated to "element item". Element items will be referred to by their [local name] in angle brackets, e.g., "the <type> element item" means the element item with the [local name] "type".

3. EncodingInstructionAssignmentList Translation

As described in the specification for ASN.X [ASN.X], the translation of an EncodingControlsection for GSER is an element item with the [local name] "GSER". The translation of the EncodingInstructionAssignmentList in such an EncodingControlsection determines the content of the <GSER> element item.

The EncodingInstructionAssignmentList for GSER is currently defined to be empty [GSEREI]; therefore, the [attributes] and [children] of the <GSER> element item are both empty.

4. EncodingInstruction Translation

The translation of an EncodingInstruction for GSER can appear in the translation of an EncodingPrefix. The translation for an EncodingPrefix is described by the specification for ASN.X.

The translation of an EncodingInstruction for GSER is the translation of the ChoiceofStringsInstruction in the EncodingInstruction (currently the only defined encoding instruction for GSER) .

\subsection{ChoiceofStringsInstruction Translation}

The translation of a ChoiceofStringsInstruction is an element item with the [local name] "choiceofstrings".

If the ChoiceofStringsInstruction has an AlternativesPrecedence, then an attribute item with the [local name] "precedence" SHALL be added to the [attributes] of the <choiceofstrings> element item. The [normalized value] of this attribute item is the white space separated list of qualified names for the expanded names [XMLNS10] of 
the NamedType instances (see [RXEREI]) corresponding to the identifiers nested in the Precedencelist in the AlternativesPrecedence.

Example

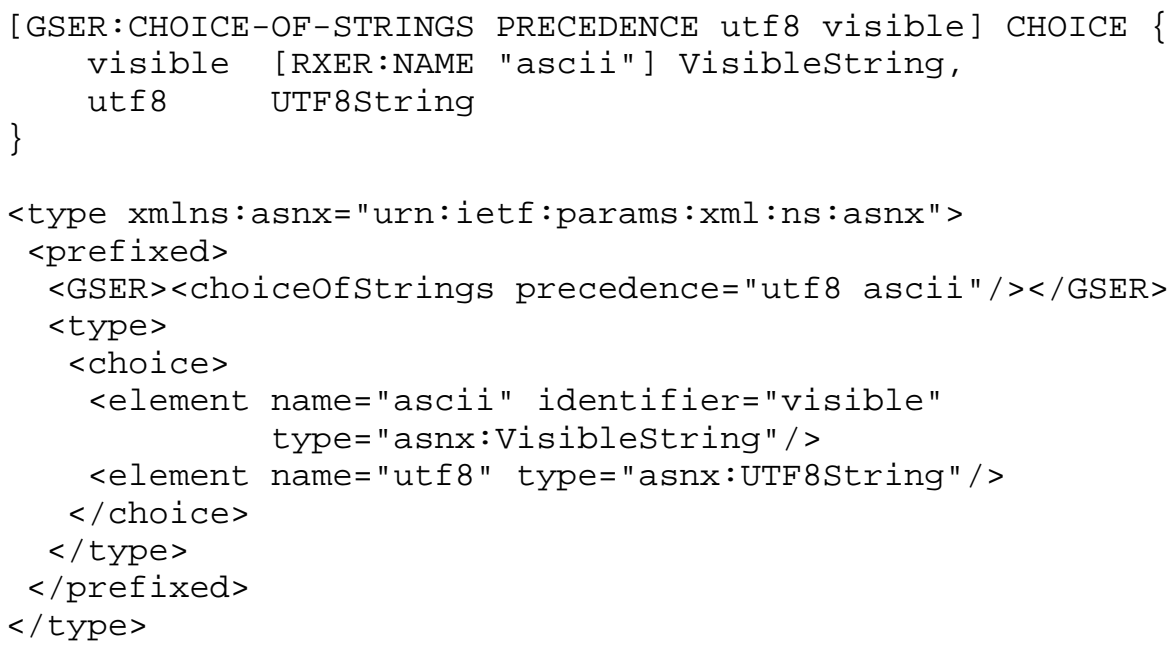

5. Security Considerations

The ASN.X translation of a GSER encoding instruction is semantically equivalent to the original GSER encoding instruction. The security considerations that apply to an application built from an original ASN.1 specification with GSER encoding instructions apply equally to an application built from the ASN.X translation of the ASN.1 specification.

See the main specification for ASN.X [ASN.X] for security considerations related to ASN.X modules.

6. Normative References

[BCP14] Bradner, S., "Key words for use in RFCs to Indicate Requirement Levels", BCP 14, RFC 2119, March 1997.

[GSER] Legg, S., "Generic String Encoding Rules (GSER) for ASN.1 Types", RFC 3641, October 2003.

[GSEREI] Legg, S., "Encoding Instructions for the Generic String Encoding Rules (GSER)", RFC 4792, January 2007. 
[RXER] Legg, S. and D. Prager, "Robust XML Encoding Rules (RXER) for Abstract Syntax Notation One (ASN.1)", RFC 4910, July 2007 .

[RXEREI] Legg, S., "Encoding Instructions for the Robust XML Encoding Rules (RXER)", RFC 4911, July 2007.

[ASN.X] Legg, S., "Abstract Syntax Notation X (ASN.X)", RFC 4912, July 2007 .

[X.680] ITU-T Recommendation X.680 (07/02) | ISO/IEC 8824-1, Information technology - Abstract Syntax Notation One (ASN.1): Specification of basic notation.

[X.680-1] ITU-T Recommendation X.680 (2002) Amendment 1 (10/03) | ISO/IEC 8824-1:2002/Amd 1:2004, Support for EXTENDED-XER.

[XML10] Bray, T., Paoli, J., Sperberg-McQueen, C., Maler, E. and F. Yergeau, "Extensible Markup Language (XML) 1.0 (Fourth Edition)", W3C Recommendation, http://www.w3.org/TR/2006/REC-xml-20060816, August 2006.

[XML11] Bray, T., Paoli, J., Sperberg-McQueen, C., Maler, E., Yergeau, F., and J. Cowan, "Extensible Markup Language (XML) 1.1 (Second Edition)", W3C Recommendation, http://www.w3.org/TR/2006/REC-xml11-20060816, August 2006.

[XMLNS10] Bray, T., Hollander, D., Layman, A., and R. Tobin, "Namespaces in XML 1.0 (Second Edition)", W3C Recommendation, http://www.w3.org/TR/2006/REC-xml-names-20060816, August 2006 .

[INFOSET] Cowan, J. and R. Tobin, "XML Information set (Second Edition)", W3C Recommendation, http: //www.w3.org/TR/2004/REC-xml-infoset-20040204, February 2004 . 
Appendix A. ASN.1 for GSER Encoding Instruction Notation

This appendix is normative.

GSER-EncodingInstructionNotation

$\{$ iso(1) identified-organization(3) dod(6)

internet(1) private(4) enterprise(1)

xmled(21472) asnx(1) module(0) gser-ei-notation(2) \}

-- Copyright (C) The IETF Trust (2007). This version of

-- this ASN.1 module is part of RFC 4913; see the RFC itself

-- for full legal notices.

$--$

-- Regarding this ASN.1 module or any portion of it, the author

-- makes no guarantees and is not responsible for any damage

-- resulting from its use. The author grants irrevocable permission

-- to anyone to use, modify, and distribute it in any way that does

-- not diminish the rights of anyone else to use, modify, and

-- distribute it, provided that redistributed derivative works do

-- not contain misleading author or version information.

-- Derivative works need not be licensed under similar terms.

DEFINITIONS

RXER INSTRUCTIONS

AUTOMATIC TAGS

EXTENSIBILITY IMPLIED : := BEGIN

IMPORTS

Precedencelist

FROM AbstractSyntaxNotation-X

$\{$ iso(1) identified-organization(3) dod(6)

internet(1) private(4) enterprise(1)

xmled(21472) asnx(1) module(0) notation(1) \}

GSER-EncodingInstruction : := [SINGULAR-INSERTIONS] CHOICE \{ choiceOfStrings GSER-ChoiceOfStringsInstruction \}

GSER-EncodingInstructionAssignmentList : := SEQUENCE \{\}

GSER-ChoiceOfStringsInstruction : := SEQUENCE \{

precedence [ATTRIBUTE] Precedencelist OPTIONAL \}

ENCODING-CONTROL RXER

SCHEMA-IDENTITY "urn:oid:1.3.6.1.4.1.21472.1.0.2" 
TARGET-NAMESPACE "urn:ietf:params:xml:ns:asnx" PREFIX "asnx" END

Appendix B. ASN.X for GSER Encoding Instruction Notation

This appendix is non-normative.

$<$ ?xml version="1.0"?>

<asnx:module xmlns:asnx="urn: ietf:params:xml:ns:asnx" name="GSER-EncodingInstructionNotation" identifier="1.3.6.1.4.1.21472.1.0.2" schemaIdentity="urn:oid:1.3.6.1.4.1.21472.1.0.2" target Namespace $=$ "urn: ietf $:$ params: $x m l: n s:$ asnx" targetPrefix="asnx" extensibilityImplied="true">

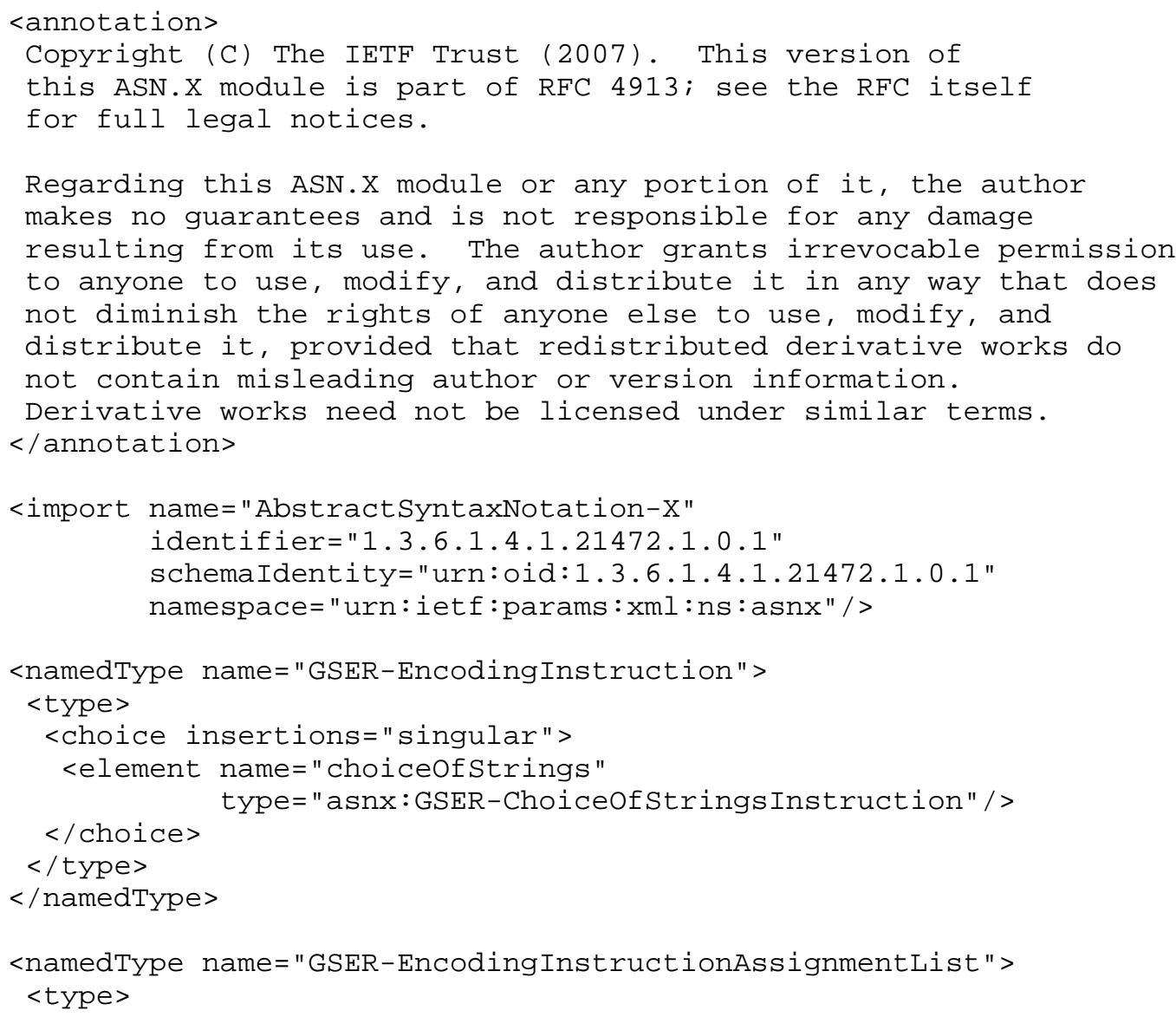




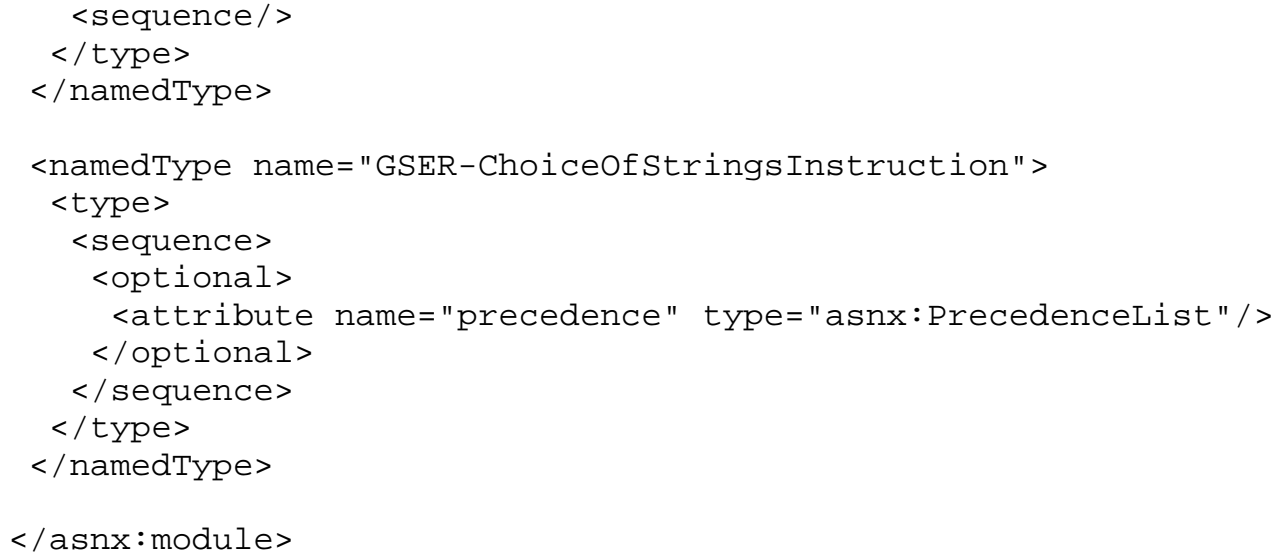

EMail: steven.legg@eb2bcom.com 
Full Copyright statement

Copyright (C) The IETF Trust (2007) .

This document is subject to the rights, licenses and restrictions contained in BCP 78, and except as set forth therein, the authors retain all their rights.

This document and the information contained herein are provided on an "AS IS" basis and THE CONTRIBUTOR, THE ORGANIZATION HE/SHE REPRESENTS OR IS SPONSORED BY (IF ANY), THE INTERNET SOCIETY, THE IETF TRUST AND THE INTERNET ENGINEERING TASK FORCE DISCLAIM ALL WARRANTIES, EXPRESS OR IMPLIED, INCLUDING BUT NOT LIMITED TO ANY WARRANTY THAT THE USE OF THE INFORMATION HEREIN WILL NOT INERINGE ANY RIGHTS OR ANY IMPLIED WARRANTIES OF MERCHANTABILITY OR FITNESS FOR A PARTICULAR PURPOSE.

Intellectual Property

The IETF takes no position regarding the validity or scope of any Intellectual property Rights or other rights that might be claimed to pertain to the implementation or use of the technology described in this document or the extent to which any license under such rights might or might not be available; nor does it represent that it has made any independent effort to identify any such rights. Information on the procedures with respect to rights in RFC documents can be found in $\mathrm{BCP} 78$ and $\mathrm{BCP} 79$.

Copies of IPR disclosures made to the IETF Secretariat and any assurances of licenses to be made available, or the result of an attempt made to obtain a general license or permission for the use of such proprietary rights by implementers or users of this specification can be obtained from the IETF on-line IPR repository at http://www.ietf.org/ipr.

The IETF invites any interested party to bring to its attention any copyrights, patents or patent applications, or other proprietary rights that may cover technology that may be required to implement this standard. Please address the information to the IETF at ietf-ipreietf.org.

Acknowledgement

Funding for the RFC Editor function is currently provided by the Internet Society. 
